\title{
Effect of the anomalous magnetic moment of quarks on magnetized QCD matter and meson spectra
}

\author{
Kun Xu®, ${ }^{1,2, *}$ Jingyi Chao $\odot,^{3}$ and Mei Huang ${ }^{1, \dagger}$ \\ ${ }^{1}$ School of Nuclear Science and Technology, University of Chinese Academy of Sciences, \\ Beijing 100049, China \\ ${ }^{2}$ Institute of High Energy Physics, Chinese Academy of Sciences, \\ Beijing 100049, People's Republic of China \\ ${ }^{3}$ College of Physics and Communication Electronics, Jiangxi Normal University, \\ Nanchang 330022, China
}

(Received 5 August 2020; accepted 17 March 2021; published 22 April 2021)

\begin{abstract}
We systematically investigate the effects made by the anomalous magnetic moment (AMM) of quark in the magnetized QCD matter, including the magnetic susceptibility, the inverse magnetic catalysis around the critical temperature and the modified neutral and or charged pion and rho meson's spectra. The dynamical AMM of quark, coupling with magnetic field, causes Zeeman splitting in the energy dispersion of quark and thus changes the magnetism properties and masses of magnetized mesons. Unfortunately, we found that the lattice results of the quark matter under magnetic fields cannot fully be explained via including the AMM interaction. It is observed that the AMM of quark reduces the dynamical quark mass and therefore induces the inverse magnetic catalysis around $T_{c}$. The neutral pion is very sensitive to the AMM term and its mass decreases with magnetic field quickly. On the contrary, the charged pion mass shows a nontrivial behavior, i.e., linearly increases with the weak and moderate magnetic fields and then saturates at strong region. For rho mesons, AMM coupling modifies the masses of neutral rho particles for all $s_{z}$ consistently, while it reduces the masses of charged rho mesons for $s_{z}=+1,0$ but enhances the mass of $s_{z}=-1$ state. The magnetic susceptibility at low temperatures can be either positive or negative with different strengths of AMM interaction.
\end{abstract}

DOI: $10.1103 /$ PhysRevD.103.076015

\section{INTRODUCTION}

Understanding properties of QCD matter under strong magnetic filed is of vital importance to further explore the interior of magnetar [1,2], neutron-star merges [3,4], noncentral heavy-ion collisions $[5,6]$, and the evolution of the early universe [7]. The study of the QCD vacuum and strongly interacting matter under external magnetic fields has attracted much attention, see reviews, e.g., Refs. [8-12]. With the presence of a magnetic field background, the strongly interacting matter shows a large number of exotic phenomena, for example, chiral magnetic effect (CME) [13-16], magnetic catalysis (MC) in the vacuum [17-19], inverse magnetic catalysis (IMC) around the critical temperature [20-22].

\footnotetext{
*xukun@mail.ihep.ac.cn

thuangmei@ucas.ac.cn
}

Published by the American Physical Society under the terms of the Creative Commons Attribution 4.0 International license. Further distribution of this work must maintain attribution to the author(s) and the published article's title, journal citation, and DOI. Funded by SCOAP .
The catalysis of the chiral symmetry breaking induced by the magnetic field, i.e., the MC effect can be easily understood from the dimension reduction. On the other hand, the IMC effect, the critical temperature of chiral phase transition decreases with growing magnetic field, which intuitively contradicts with the MC effect and still remains as a puzzle, though there have been many works in the literature trying to explain the IMC by considering neutral pion fluctuations [23], chirality imbalance [24], running coupling constant [25]. Recently, lattice calculations present more interesting and novel properties of magnetized QCD matter, such as the charged pion mass shows a nonmonotonic magnetic field dependent behavior [26], and magnetized matter exhibits diamagnetism (negative susceptibility) at low temperature and paramagnetism (positive susceptibility) at high temperature [27,28].

Magnetic fields modify the spectrum of charged particles. The point particle approximation gives the charged pion masses $m_{\pi^{ \pm}}^{2}(B)=m_{\pi^{ \pm}}^{2}(B=0)+e B$ increasing linearly along the magnetic field and the neutral pion mass keeps as a constant. Calculation in the effective quark model, e.g., the Namu-Jona-Lasinio (NJL) model which taking into account of quark magnetization, modifies the 
linear slope of charged pion and shows point-approximation results for pion mass spectra [29-39]. In NJL model, mesons are treated as quantum fluctuations in random phase approximation (RPA), which are created via a summation of an infinite number of quark loops [32,33,36,37,40-42]. However, with presence of magnetic field, the Schwinger phase appears in each quark propagator [43]. For neutral pion, the Schwinger phases cancel out for each loop, while they do not for charged pion. In Refs. [36,37] the authors employed the Ritus eigenfunction method in the two-flavor NJL model, which allows us to properly take into account the presence of Schwinger phases in the quark propagators. They found that in the region $e B \sim 0-1.5 \mathrm{GeV}^{2}$, neutral pion mass decreases slightly while charged pion mass steadily increases. And in Ref. [32], it is found that the charged pion becomes much heavier in the magnetic field and is sensitive to the field strength, while the neutral pion still keeps as a NambuGoldstone particle in the region around $e B \sim 0-0.4 \mathrm{GeV}^{2}$. Reference [34] shows that neutral pion mass first decreases and then increases with magnetic field, which is consistent with lattice result in Refs. [44,45]. Similar results are also obtained in another lattice calculation Ref. [46]. In Ref. [20], charged pion mass has been non-perturbatively computed, and it found that the masses of $\pi^{ \pm}$increase with magnetic field in the region of $e B \sim 0-0.3 \mathrm{GeV}^{2}$, however, for $e B>0.3 \mathrm{GeV}^{2}$, charged pion masses do not show a increase trend with magnetic field. Recent lattice calculation in Ref. [26] shows that the neutral pion mass decreases with the magnetic field while the charged pion and kaon present non-monotonic behaviors, which first increases linearly and then decreases as magnetic field increasing, and all these masses show a saturation at $e B \gtrsim 2.5 \mathrm{GeV}^{2}$, which are quite different from pointparticle approximation and previous results from effective models. It is worth mentioning that lattice results in $[20,46]$ show a saturation behavior for charged pion and no decreasing with magnetic field is observed at strong magnetic field.

The remaining IMC puzzle and recently discovered properties of magnetized matter attract our renewed interest to revisit $Q C D$ vacuum and matter under external magnetic field and to find the underlying mechanism for these properties. It is known that the dynamical chiral symmetry broken is one of the most significant feature of QCD, where quark obtains a dynamical mass. It has been found that the anomalous magnetic moments (AMM) of quarks can also be generated dynamically like dynamical quark mass [47-49]. In Ref. [48] the authors explained how dynamical chiral symmetry breaking produces a dressed light-quark with a momentum-dependent anomalous chromomagnetic moment as well as an anomalous electromagnetic moment to fulfill the Ward identity. In Ref. [47], the authors considered one-flavor NJL model with a new channel $\sim\left(\bar{\psi} \Sigma^{3} \psi\right)^{2}+\left(\bar{\psi} i \gamma^{5} \Sigma^{3} \psi\right)^{2}$ in the presence of magnetic field, and they found that the chiral condensate and new condensate $\left\langle\bar{\psi} i \gamma^{1} \gamma^{2} \psi\right\rangle$, which corresponding to AMM for quarks, differ from zero at vacuum with magnetic field simultaneously. A two-flavor NJL model with tensor channel was also investigated in Refs. [50,51], and similar results were obtained. In Refs. [49,52], the authors investigated the nonperturbative generation of an AMM for massless fermions under external magnetic field, and they proved that the phenomenon of magnetic catalysis of chiral symmetry breaking is also responsible for the generation of the dynamical AMM. Thus, once the quarks acquire a dynamical mass, they should also acquire a dynamical AMM [47-49,52,53].

There have been several studies working on how AMM of quarks influence QCD phase diagram as well as mesonic properties [25,54-60]. For example, in Ref. [56], the authors used two-flavor NJL model with AMM and found that the critical temperature for chiral transition decreases with the external magnetic field, while a sudden jump for pion mass at and above the Mott transition temperature appears when the AMM of the quarks are taken into consideration. And Ref. [54] found that the inverse magnetic catalysis occurs for large enough AMM coupling. In Ref. [25], the authors considered NJL model with dynamical inducing AMM and found that with magnetic-dependent coupling constants, the scenario of IMC can be produced.

In this paper, we focus on the dynamical quark mass and meson mass, e.g., pion and rho, in the presence of magnetic field with anomalous magnetic moments of quarks. This paper is organized as follows: in Sec. II we introduce the two-flavor NJL model with AMM in the external magnetic field, and investigate the effect of AMM on dynamical quark mass and the magnetism property. Then we investigate pion and rho masses as functions of magnetic fields with different AMM in Sec. III. Finally, we discuss the results in Sec. IV.

\section{MODEL SETUP}

We choose two-flavor NJL model including the AMM of quarks in the presence of magnetic field, whose Lagrangian takes form of $[54,56,58]$ :

$$
\begin{aligned}
\mathcal{L}= & \bar{\psi}\left(i \gamma^{\mu} D_{\mu}-m_{0}+\kappa_{f} q_{f} F_{\mu \nu} \sigma^{\mu \nu}\right) \psi \\
& +G_{S}\left\{(\bar{\psi} \psi)^{2}+\left(\bar{\psi} i \gamma^{5} \vec{\tau} \psi\right)^{2}\right\} \\
& -G_{V}\left\{\left(\bar{\psi} \gamma^{\mu} \vec{\tau} \psi\right)^{2}+\left(\bar{\psi} \gamma^{\mu} \gamma^{5} \vec{\tau} \psi\right)^{2}\right\} .
\end{aligned}
$$

Here $\psi$ are two-flavor quark fields $\psi=(u, d)^{T}, m_{0}$ is current mass which assumed to be same for both flavors, i.e., $m_{u}=m_{d}=m_{0}$. The covariant derivative $D_{\mu}=$ $\partial_{\mu}-i q_{f} A_{\mu}$ with $q_{f}$ being the electric charge of quarks, $A_{\mu}$ being the abelian gauge field and field strength $F_{\mu \nu}=\partial_{\mu} A_{\nu}-\partial_{\nu} A_{\mu}$. Without loss of generality, we choose external uniform magnetic field along $z$-direction, which 
led to $A_{\mu}=\{0,0, B x, 0\}$. The term $\bar{\psi} \kappa_{f} q_{f} F_{\mu \nu} \sigma^{\mu \nu} \psi$, with $\sigma^{\mu \nu}=\frac{i}{2}\left[\gamma^{\mu}, \gamma^{\nu}\right]$, brings the contribution of AMM. $\kappa_{f}$ is defined as $\kappa_{f}=\alpha_{f} \mu_{B}$ with Bohr magneton $\mu_{B}=\frac{e}{2 M}$ and constituent quark mass $M$, which will define below. At oneloop level we have $\alpha_{f}=\frac{\alpha_{e} q_{f}^{2}}{2 \pi}$ with $\alpha_{e}=\frac{1}{137}$, which is the electromagnetic fine structure constant. However, to study how AMM term affects quark mass and meson mass, we treat $\kappa_{f}$ as a free and flavor-independent parameter, e.g., $\kappa_{u}=\kappa_{d}=\kappa$. Besides, $G_{S}$ and $G_{V}$ are the coupling constants for (pseudo-)scalar and (pseudo-)vector interaction channels, respectively. After mean-field approximation, the Lagrangian is hence given by:

$$
\begin{aligned}
\mathcal{L}= & -\frac{\left(M-m_{0}\right)^{2}+\pi^{a} \pi^{a}}{4 G_{S}}+\frac{V_{\mu}^{a} V^{\mu, a}+A_{\mu}^{a} A^{\mu, a}}{4 G_{V}} \\
& +\bar{\psi}\left(i \gamma^{\mu} D_{\mu}-M+\kappa q_{f} B \sigma^{12}\right) \psi,
\end{aligned}
$$

where we define:

$$
\begin{aligned}
& M=m_{0}-2 G_{S}\langle\bar{\psi} \psi\rangle, \quad \vec{\pi}=-2 G_{S}\left\langle\bar{\psi} i \gamma^{5} \vec{\tau} \psi\right\rangle, \\
& V_{\mu}^{a}=-2 G_{V}\left\langle\bar{\psi} \gamma_{\mu} \tau^{a} \psi\right\rangle, \quad A_{\mu}^{a}=-2 G_{V}\left\langle\bar{\psi} \gamma_{\mu} \gamma^{5} \tau^{a} \psi\right\rangle .
\end{aligned}
$$

It is well known that NJL model is nonrenormalized, thus regularization scheme is necessary for extracting finite numerical results. In this work, a soft cutoff is applied for momentum integration and Landau level summation during the numerical calculation:

$$
\frac{\left|q_{f} B\right|}{2 \pi} \sum_{n} \int \frac{d p_{z}}{2 \pi} \rightarrow \frac{\left|q_{f} B\right|}{2 \pi} \sum_{n} \int \frac{d p_{z}}{2 \pi} f_{\Lambda}\left(p_{z}, n\right)
$$

with

$$
f_{\Lambda}\left(p_{z}, n\right)=\frac{\Lambda^{10}}{\Lambda^{10}+\left(p_{z}^{2}+2 n\left|q_{f} B\right|\right)^{5}} .
$$

There are four parameters in total: current quark mass $m_{0}$, three-momentum cutoff parameter $\Lambda$ in Eq. (5), (pseudo-) scalar/(pseudo-)vector coupling constant $G_{S}$ and $G_{V}$. These parameters are determined by fitting to experimental data at zero temperature and vanishing magnetic field. In this work, we have used two sets of parameters: (I), $m_{0}=5 \mathrm{MeV}, \Lambda=624.18 \mathrm{MeV}$ and $G_{S} \Lambda^{2}=2.014$, which corresponds to pion decay constant $f_{\pi}=93 \mathrm{MeV}$, pion mass $m_{\pi}=135.6 \mathrm{MeV}$ as well as the quark condensate $\langle\bar{\psi} \psi\rangle=-(251.8 \mathrm{MeV})^{3}$. This set of parameters is used to investigate both dynamical quark and pion masses; (II), $m_{0}=5 \mathrm{MeV}, \Lambda=582 \mathrm{MeV}, G_{S} \Lambda^{2}=2.388$ and $G_{V} \Lambda^{2}=1.73$, which are chosen in a such way that $m_{\pi}=140 \mathrm{MeV}, m_{\rho}=768 \mathrm{MeV}$ with $M=458 \mathrm{MeV}$ at zero temperature as used in Ref. [29,41]. Here, the quark condensate $\langle\bar{\psi} \psi\rangle=-(267 \mathrm{MeV})^{3}$. In particular, the dynamical generated quark mass $M$ resulting from parameter set II is deliberately chosen to be such large to avoid the decay process $\rho \rightarrow q \bar{q}$ at zero temperature. We will apply this set to study rho meson.

\section{A. Dispersion relation for fermions with AMM}

In this part, we derive the dispersion relation for positive charged fermion $\psi$ with charge $q$, dynamical mass $M$ and the anomalous magnetic moment $\kappa$ in the presence of homogeneous magnetic field $B$ where the Dirac equation is given by

$$
\left(i \gamma^{\mu} D_{\mu}-M+\frac{1}{2} \kappa q \sigma^{\mu \nu} F_{\mu \nu}\right) \psi=0
$$

Similar with Eq. (1), we assume that the magnetic field orients to $z$ direction. To simplify following writing, we set $T=\kappa q B$, and the Dirac equation becomes:

$$
\left(i \gamma^{\mu} D_{\mu}-M+T \sigma^{12}\right) \psi=0
$$

where $\sigma^{12}=i \gamma^{1} \gamma^{2}$. For this equation, the general solution of $\psi$ has form of:

$$
\psi=\mathrm{e}^{-i E t}\left(\begin{array}{l}
\phi \\
\chi
\end{array}\right)
$$

where $\phi$ and $\chi$ are the two-component spinors. Inserting Eq. (8) into Eq. (7), where chiral representations of the $\gamma$ matrices are used, we obtain the coupled equations for $\phi$ and $\chi$ :

$$
\begin{aligned}
& \left(M-T \sigma^{3}\right) \phi-(E+i \vec{\sigma} \cdot \vec{D}) \chi=0 \\
& (E-i \vec{\sigma} \cdot \vec{D}) \phi-\left(M-T \sigma^{3}\right) \chi=0
\end{aligned}
$$

Eliminating $\chi$ from Eq. (9) and Eq. (10), $\phi$ is govern by:

$$
\begin{aligned}
\hat{A} \phi= & \left\{\left(M-T \sigma^{3}\right)\left(M^{2}-T^{2}\right)\right. \\
& \left.-(E+i \vec{\sigma} \cdot \vec{D})\left(M+T \sigma^{3}\right)(E-i \vec{\sigma} \cdot \vec{D})\right\} \phi=0 .
\end{aligned}
$$

Obviously, $\phi$ is a two-component spinor while $\hat{A}$ is a $2 \times 2$ matrix. The elements of $\hat{A}$ are listed as following:

$$
\begin{aligned}
\hat{A}_{12}= & 2 T\left(E+i D_{3}\right)\left(i D_{1}+D_{2}\right), \\
\hat{A}_{21}= & -2 T\left(E-i D_{3}\right)\left(i D_{1}-D_{2}\right), \\
\hat{A}_{11}= & (M+T)(M-T)^{2}-(M+T)\left(E^{2}+D_{3}^{2}\right) \\
& +(M-T)\left(i D_{1}+D_{2}\right)\left(i D_{1}-D_{2}\right), \\
\hat{A}_{22}= & (M-T)(M+T)^{2}-(M-T)\left(E^{2}+D_{3}^{2}\right) \\
& +(M+T)\left(i D_{1}-D_{2}\right)\left(i D_{1}+D_{2}\right) .
\end{aligned}
$$


To determine the form of $\phi$, we apply the function $f_{k}(x)$ formed as below:

$$
f_{k}(x)=c_{k} \mathrm{e}^{-\frac{1}{2}\left(\frac{x}{l}-p_{y} l\right)^{2}} H_{k}\left(\frac{x}{l}-p_{y} l\right) \mathrm{e}^{i\left(\xi_{f} p_{y} y+p_{z} z\right)},
$$

where $H_{k}(x)$ is the Hermite polynomials, $c_{k}$ is the normalized constant, with $l=1 / \sqrt{|q B|}, \quad \xi_{f}=\operatorname{sgn}(q)$, and $f_{-1}=0$ at the lowest state. After a tedious calculation, the following relations for $q>0$ are obtained:

$$
\begin{aligned}
& \left(i D_{1}+D_{2}\right) f_{k}=-i \sqrt{|q B|} c_{k, k+1} f_{k+1}, \\
& \left(i D_{1}-D_{2}\right) f_{k}=2 i k \sqrt{|q B|} c_{k, k-1} f_{k-1},
\end{aligned}
$$

where $c_{n, m}=c_{n} / c_{m}$. In fact, the operators $i D_{1}+D_{2}$ and $i D_{1}-D_{2}$ are creation and annihilation operators, respectively. And then the general form of $\phi$ is straightforward, written as:

$$
\phi_{k}(x)=\left(\begin{array}{c}
f_{k}(x) \\
f_{k-1}(x)
\end{array}\right)
$$

Inserting Eq. (12) and Eq. (16) into Eq. (11), we obtain two decoupled equations:

$$
\begin{aligned}
& \left\{(M+T)(M-T)^{2}-(M+T)\left(E^{2}-p_{z}^{2}\right)\right. \\
& \quad+2 k(M-T)|q B|\} f_{k} \\
& \quad-2 i T\left(E-p_{z}\right) \sqrt{|q B|} c_{k-1, k} f_{k}=0, \\
& \left\{(M-T)(M+T)^{2}-(M-T)\left(E^{2}-p_{z}^{2}\right)\right. \\
& \quad+2 k(M+T)|q B|\} f_{k-1} \\
& \quad-2 i T\left(E+p_{z}\right) \cdot 2 k \sqrt{|q B|} c_{k, k-1} f_{k-1}=0 .
\end{aligned}
$$

Combining the above two equations with relations of Eq. (14) and Eq. (15), the dispersion relation for positive-charged fermion with spin-s is extracted as:

$$
E_{k}^{2}= \begin{cases}p_{z}^{2}+\left\{\sqrt{M^{2}+2 k|q B|}-s T\right\}^{2}, & \text { if } k \geq 1 \\ p_{z}^{2}+(M-T)^{2}, & \text { if } k=0\end{cases}
$$

A similar relation for negative charged fermion can be obtained, however, we will not repeat here. Finally, putting back $T=\kappa q B$, the dispersion relation for fermions with charge $q$, spin- $s$ and AMM $\kappa$ in the presence of magnetic field is

$E_{k}^{2}=p_{z}^{2}+\left\{\sqrt{M^{2}+(2 k+1-s \xi)|q B|}-s \kappa q B\right\}^{2}$,

where $s= \pm 1$ is for spin-up and spin-down, respectively. From this dispersion relation, we can see that the lowest

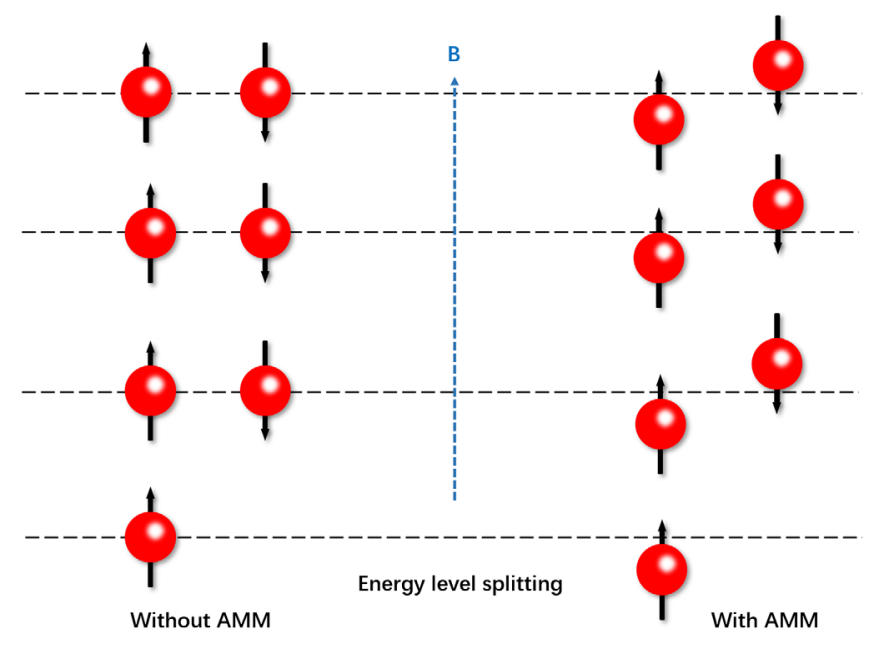

FIG. 1. Energy levels of positive charged fermion for the case with (right) and without (left) AMM in the presence of magnetic field.

Landau levels (LLL) for both spin-up/down positive/negative charged quarks have the form of

$E_{0}^{2}=p_{z}^{2}+\left(M-\kappa\left|q_{f}\right| B\right)^{2}, \quad u^{\uparrow}, \bar{d}^{\uparrow}$

$E_{0}^{2}=p_{z}^{2}+\left(M-\kappa\left|q_{f}\right| B\right)^{2}, \quad \bar{u}^{\downarrow}, d^{\downarrow}$

$E_{0}^{2}=p_{z}^{2}+\left(\sqrt{M^{2}+2\left|q_{f} B\right|}+\kappa\left|q_{f}\right| B\right)^{2}, \quad \bar{u}^{\uparrow}, d^{\uparrow}$

$E_{0}^{2}=p_{z}^{2}+\left(\sqrt{M^{2}+2\left|q_{f} B\right|}+\kappa\left|q_{f}\right| B\right)^{2} \cdot \quad u^{\downarrow}, \bar{d}^{\downarrow}$

Therefore, for two-flavor quark system, for $\kappa>0$, the lowest energy is occupied by spin-up positive-charged fermions $u^{\uparrow}, \bar{d}^{\uparrow}$ and spin-down negative-charged fermions $\bar{u}^{\downarrow}, d^{\downarrow}$. For higher excitations, either positive or negative charged fermion, the energy spectrum exhibit Zeeman splittings $(s= \pm 1)$, which can be seen clearly in Fig. 1 .

\section{B. Inverse magnetic catalysis with AMM}

From the dispersion relation Eq. (21) in the LLL, e.g., $E_{0}^{2}=p_{z}^{2}+\left(M-\kappa\left|q_{f}\right| B\right)^{2}$, we can see that AMM definitely reduces the dynamical quark mass. We numerically investigate the total effect of AMM on the dynamical quark mass, following Refs. [40,54,56,58], we obtain the oneloop level effective potential at zero baryon chemical potential and finite temperature:

$$
\begin{aligned}
\Omega= & \frac{\left(M-m_{0}\right)^{2}}{4 G_{S}}-N_{c} \sum_{f} \frac{\left|q_{f} B\right|}{2 \pi} \sum_{n} \sum_{s= \pm 1} \int \frac{d p_{z}}{2 \pi} E_{n, f, s} \\
& -2 N_{c} T \sum_{f} \frac{\left|q_{f} B\right|}{2 \pi} \sum_{n} \sum_{s= \pm 1} \int \frac{d p_{z}}{2 \pi} \ln \left(1+\mathrm{e}^{-\frac{E_{n, f, s}}{T}}\right) .
\end{aligned}
$$


Here only scalar channel is considered for the study of the dynamical quark mass, which is obtained by solving the gap equation:

$$
\frac{\partial \Omega}{\partial M}=0
$$

and in explicit manner:

$$
\begin{aligned}
\frac{M-m_{0}}{2 G_{S}}= & N_{c} \sum_{f} \frac{\left|q_{f} B\right|}{2 \pi} \sum_{n} \sum_{s= \pm 1} \int \frac{d p_{z}}{2 \pi}\left\{1-2\left(1+\mathrm{e}^{\frac{E_{n, f, s}}{T}}\right)^{-1}\right\} \\
& \times \frac{M}{E_{n, f, s}}\left(1-\frac{s \kappa q_{f} B}{M_{n}}\right) .
\end{aligned}
$$

$M=m_{0}+\sigma$ is quark's dynamical mass with

$$
\begin{aligned}
\sigma= & 2 G_{S} N_{c} \sum_{f} \frac{\left|q_{f} B\right|}{2 \pi} \sum_{n} \sum_{s \pm 1} \int \frac{d p_{z}}{2 \pi}\left\{1-2\left(1+\mathrm{e}^{\frac{E_{n, f, s}}{T}}\right)^{-1}\right\} \\
& \times \frac{M}{E_{n, f, s}}\left(1-\frac{s \kappa q_{f} B}{M_{n}}\right)
\end{aligned}
$$

where $\quad M_{n}=\sqrt{M^{2}+\left(2 n+1-s \xi_{f}\right)\left|q_{f} B\right|}-s \kappa q_{f} B$. In strong magnetic field region, we can apply LLL approximation and then the gap equation at zero temperature becomes:

$$
\begin{aligned}
M & =m_{0}+2 G_{S} N_{c} \sum_{f} \frac{\left|q_{f} B\right|}{2 \pi} \int \frac{d p_{z}}{2 \pi} \frac{1}{E_{0}}\left(1-\frac{\kappa\left|q_{f} B\right|}{M}\right) \\
& \simeq m_{0}+2 G_{S} N_{c} \sum_{f} \frac{\left|q_{f} B\right|}{2 \pi} \int \frac{d p_{z}}{2 \pi} \frac{1}{E}\left(1-\lambda \kappa+\left(\kappa^{2}\right)\right),
\end{aligned}
$$

where $E=\sqrt{p_{z}^{2}+M^{2}}$ and $\lambda=\frac{p_{z}^{2}}{M\left(M^{2}+p_{z}^{2}\right)}$. In the second line we take a Taylor expansion for small $\kappa$. It is obviously seen that a none-zero $\kappa$ reduces the quark mass $M$ in the strong magnetic field region.

The numerical results of the dynamical quark mass as a function of the magnetic field with different AMMs are shown in Figs. 2 and 3, where $\kappa$ is treated as either a constant or proportional to $\sigma$. Note here that $\sigma$ is solved from Eq. (25).

(1) We first treat $\kappa_{u}=\kappa_{d}=\kappa$ as a free constant and calculate the dynamical quark mass as a function of the magnetic field at zero temperature with different $\kappa$. The numerical results are shown in Fig. 2. It is observed clearly that the MC effect is competing with the mass reducing effect from the AMM. For small value of $\kappa \lesssim 0.6 \mathrm{GeV}^{-1}$, the dynamical quark mass $M_{q}$ increases with magnetic field, known as MC. But, the AMM explicitly reduces dynamical quark mass as shown in Fig. 2, which is consistent with the dispersion relation rendering in Eq. (21).

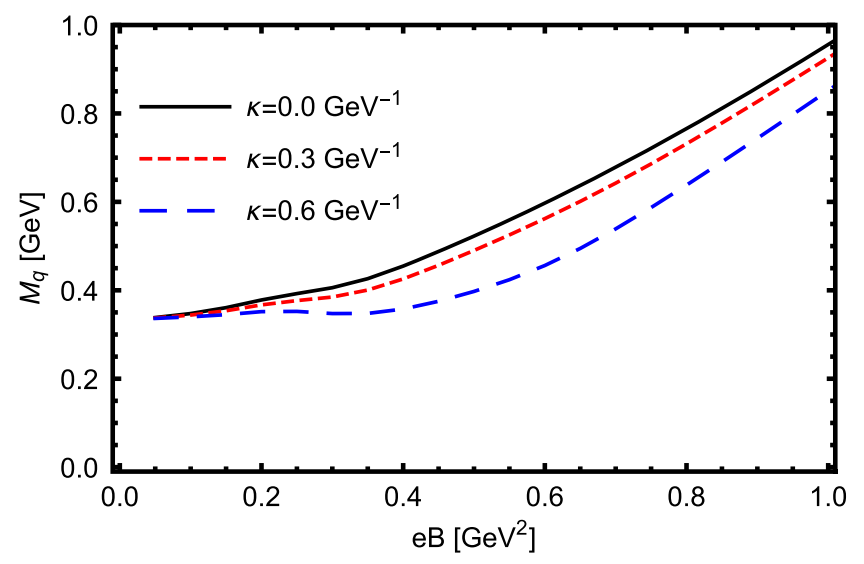

FIG. 2. Dynamical quark mass as a function of magnetic field with different constant $\kappa$ at zero temperature.

For larger $\kappa$, the mass reducing effect will exceed the MC effect in some magnetic field region, and the behavior becomes quite complicated. Therefore, in the following calculations, we will focus on small values of constant $\kappa$.

(2) Then we take $\kappa$ proportional to the quark condensate $\kappa=v \sigma$ with ratio $v$ and $\sigma$ solved from Eq. (25). The corresponding results for dynamical quark masses at zero temperature are shown in Fig. 3. It is seen that when the ratios $v$ are small, as shown by the red solid line and green dashed line, $M_{q}$ increases with magnetic field monotonically and the MC effect overcomes the mass reducing effect triggered by the AMM. When $v$ is large enough, the AMM's mass reducing effect dominates thus one can see that the dynamical quark mass decreases smoothly with the increasing magnetic field. We also calculate the dynamical quark masses at finite temperatures, under a fixed magnetic field $e B=0.5 \mathrm{GeV}^{2}$, as shown in Fig. 4(a). Ones find out that a larger $v$ not only reduces the dynamical quark mass, but also

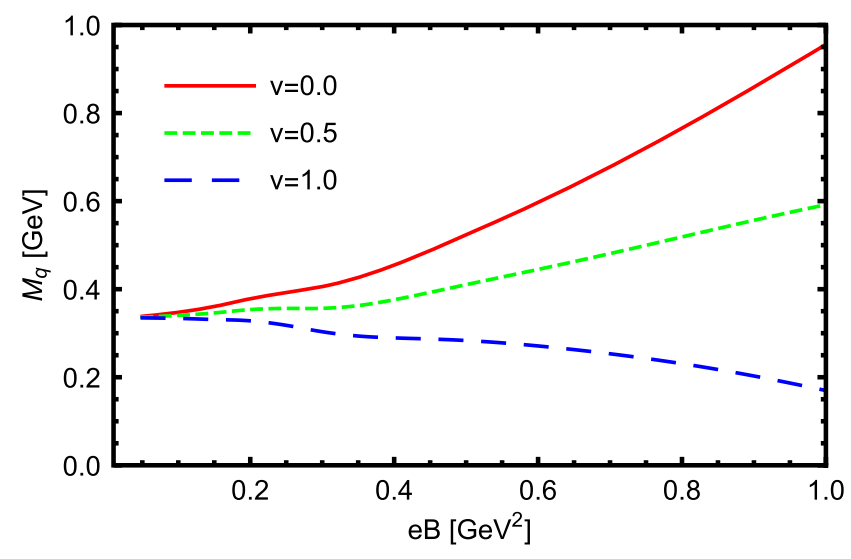

FIG. 3. Dynamical quark mass as a function of magnetic field with $\kappa=v \sigma$ at zero temperature. 


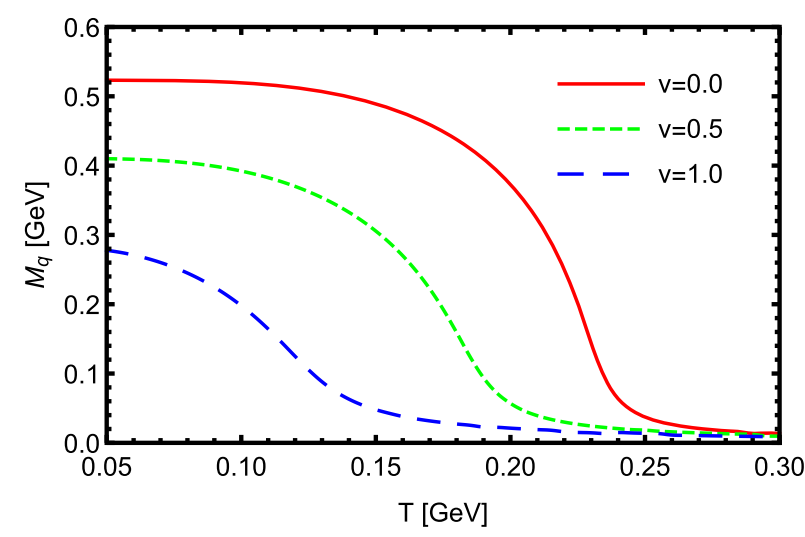

(a)

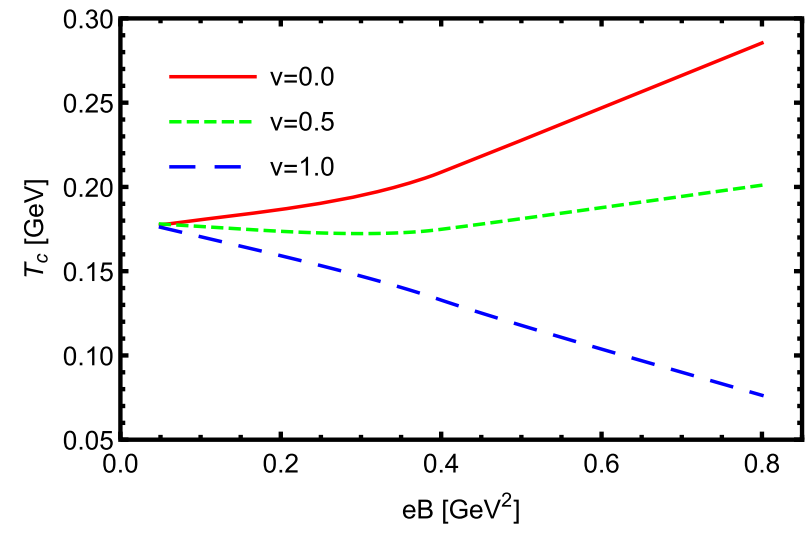

(b)

FIG. 4. (a): Dynamical quark mass $M_{q}$ as a function of temperature with $\kappa=v \sigma$ at a fixed magnetic field $e B=0.5 \mathrm{GeV}{ }^{2}$. (b): critical temperature $T_{c}$ of chiral transition aas a function of magnetic field. With proper $v$, Inverse Magnetic Catalysis can be obtained (e.g., blue dashed line).

suppresses the critical temperature $T_{c}$, which is determined through $T_{c}=\left(-\partial M_{q} / \partial T\right)$. $T_{c}$ as functions of the magnetic fields under different $v$ are plotted in Fig. 4(b). For a small $v$, conventional MC shows up, i.e., $T_{c}$ increases with the magnetic field, however, when $v$ is large, for example, shown by the blue dashed line for $v=1, T_{c}$ decreases with the magnetic field, which indicates the IMC.

\section{Magnetic susceptibility with AMM}

Recently, lattice calculations show more interesting and novel properties of magnetized QCD matter, e.g., magnetized matter exhibits diamagnetism (negative susceptibility) at low temperatures and paramagnetism (positive susceptibility) at high temperatures [27,28]. From the above dispersion relation, one observes that the AMM of quarks causes Zeeman splitting in the dispersion relation which plays an important role in the magnetism properties. We show the numerical results for magnetic susceptibility induced by the AMM. The magnetic susceptibility is defined as:

$$
\chi=-\left.\frac{\partial^{2} \Omega}{\partial(e B)^{2}}\right|_{e B=0},
$$

and $\chi_{0}(T)=\chi(T)-\chi(T=0)$. The numerical results are shown in Fig. 5, where soft cutoff is applied. At high temperatures, there is no doubt that the magnetized matter shows paramagnetism with $\chi_{0}>0$. However, at low temperatures, a negative $\kappa$ gives negative susceptibility, i.e., which indicates the diamagnetism of magnetized QCD matter, while a positive $\kappa$ gives positive susceptibility, i.e., the paramagnetism of magnetized QCD matter. Therefore, the diamagnetism property cannot be explained by considering the AMM of quarks.

\section{MESON SPECTRA WITH AMM}

Next, we investigate meson spectra with the AMM. In the NJL model, mesons are regarded as $q \bar{q}$ bound states or resonances, which are obtained from the quark-antiquark scattering amplitudes [40-42], as shown in Fig. 6. The full propagator of meson is expressed to leading order in $1 / N_{c}$ as an infinite sum of quark-loop chains under the random phase approximation. Following the procedure given in Ref. [9], the quark propagator in the Landau level representation is given by

$$
S(x, y)=\mathrm{e}^{i \Phi_{f}(x, y)} \int \frac{d^{4} q}{(2 \pi)^{4}} \mathrm{e}^{-i(x-y) q} \tilde{S}(q),
$$

where the Schwinger phase $\Phi_{f}(x, y)=q_{f}\left(x^{1}+y^{1}\right)\left(x^{2}-\right.$ $\left.y^{2}\right) / 2$ breaks the translation invariant while $\widetilde{S}(q)$ is translation-invariant and takes the form of:

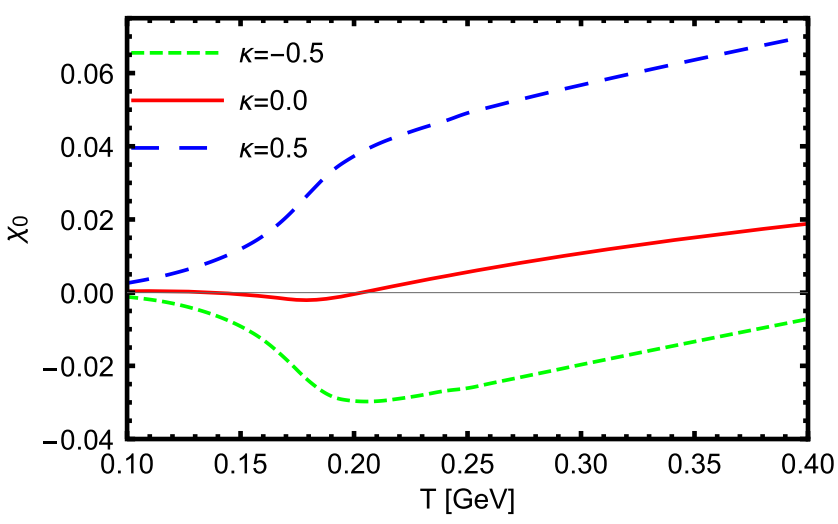

FIG. 5. Magnetic susceptibility with different constant $\kappa$. A soft cutoff is applied during the numerical calculation. 

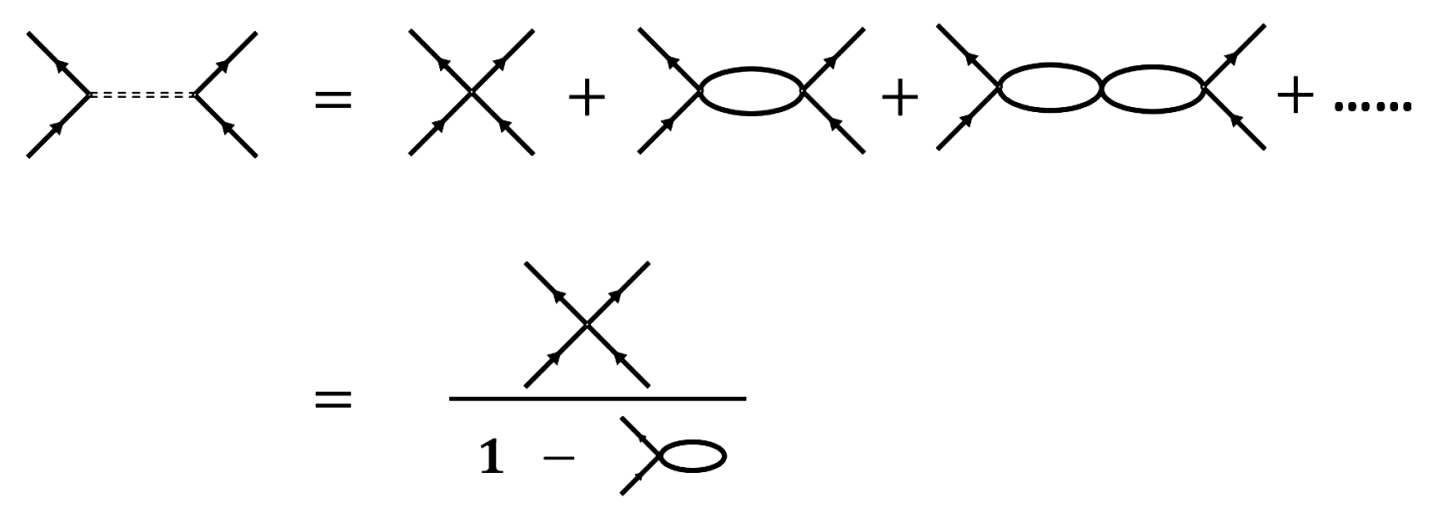

FIG. 6. Meson propagator under the random phase approximation in the NJL model.

$\widetilde{S}_{f}(k)=i \exp \left(-\frac{\mathbf{k}_{\perp}^{2}}{\left|q_{f} B\right|}\right) \sum_{n=0}^{\infty}(-1)^{n} \frac{D_{n}\left(q_{f} B, k\right) F_{n}\left(q_{f} B, k\right)}{A_{n}\left(q_{f} B, k\right)}$.

Here,

$F_{n}\left(q_{f} B, k\right)=\left(\kappa q_{f} B-k^{0} \gamma^{3} \gamma^{5}+k^{3} \gamma^{0} \gamma^{5}\right)^{2}-M^{2}-2 n\left|q_{f} B\right|$,

and

$$
\begin{aligned}
D_{n}\left(q_{f} B, k\right)= & \left(k^{0} \gamma^{0}-k^{3} \gamma^{3}+M+\kappa q_{f} B \sigma^{12}\right) \\
& \times\left[\left(1-i \gamma^{1} \gamma^{2} \xi_{f}\right) L_{n}\left(2 \frac{\mathbf{k}_{\perp}^{2}}{\left|q_{f} B\right|}\right)\right. \\
& \left.-\left(1+i \gamma^{1} \gamma^{2} \xi_{f}\right) L_{n-1}\left(2 \frac{\mathbf{k}_{\perp}^{2}}{\left|q_{f} B\right|}\right)\right] \\
& +4\left(k^{1} \gamma^{1}+k^{2} \gamma^{2}\right) L_{n-1}^{1}\left(2 \frac{\mathbf{k}_{\perp}^{2}}{\left|q_{f} B\right|}\right),
\end{aligned}
$$

where $\xi_{f}=\operatorname{sign}\left(q_{f} B\right), L_{n}^{\alpha}$ are the generalized Laguerre polynomials, $L_{n}=L_{n}^{0}$ and the denominator:

$$
\begin{aligned}
A_{n}\left(q_{f} B, k\right)= & \Pi_{s= \pm 1}\left\{\left(\kappa q_{f} B+s \sqrt{\left(k^{0}\right)^{2}-\left(k^{3}\right)^{2}}\right)^{2}\right. \\
& \left.-M^{2}-2 n\left|q_{f} B\right|\right\} .
\end{aligned}
$$

With RPA approximation, the composite $\pi$ propagator is written as:

$$
D_{\pi}\left(q^{2}\right)=\frac{2 G_{S}}{1-2 G_{S} \Pi_{\pi}\left(q^{2}\right)},
$$

where $\Pi_{\pi}$ is the one loop polarization function for pion:

$$
\Pi_{\pi}\left(q^{2}\right)=i \int \frac{d^{4} k}{(2 \pi)^{4}} \operatorname{Tr}\left[\tau^{a} i \gamma^{5} \widetilde{S}(k) \tau^{b} i \gamma^{5} \widetilde{S}(p)\right],
$$

with $q=k-p$. For neutral pion, the Pauli matrices take $\tau^{a}=\tau^{3}, \tau^{b}=\tau^{3}$ while for charged pion they take $\tau^{a}=\tau^{ \pm}$, $\tau^{b}=\tau^{\mp}$ with definition:

$$
\tau^{ \pm}=\frac{1}{\sqrt{2}}\left(\tau^{1} \mp i \tau^{2}\right)
$$

$\widetilde{S}(q)$ is the translation-invariant part of quark propagator in momentum space and its explicit expression has given in Eq. (29). Extracting from the pole of propagator $D_{\pi}\left(q^{2}\right)$, the corresponding meson mass can be obtained by solving:

$$
1-2 G_{S} \Pi_{\pi}\left(q^{2}=m_{\pi}^{2}\right)=0 .
$$

It is similar for computing the mass of the vector rho meson via corresponding one loop polarization function $\Pi_{V}^{\mu \nu}$ :

$$
\Pi_{V}^{\mu \nu}\left(q^{2}\right)=i \int \frac{d^{4} k}{(2 \pi)^{4}} \operatorname{Tr}\left[\tau^{a} \gamma^{\mu} \widetilde{S}(k) \tau^{b} \gamma^{\nu} \widetilde{S}(p)\right]
$$

And it is easily proved that the polarization function for charged rho has following structure:

$$
\Pi_{ \pm}^{\mu \nu}=\left(\begin{array}{cccc}
\Pi_{ \pm}^{00} & 0 & 0 & 0 \\
0 & \Pi_{ \pm}^{11} & \Pi_{ \pm}^{12} & 0 \\
0 & \Pi_{ \pm}^{21} & \Pi_{ \pm}^{22} & 0 \\
0 & 0 & 0 & \Pi_{ \pm}^{33}
\end{array}\right)
$$

where $\Pi_{ \pm}^{11}=\Pi_{ \pm}^{22}$ and $\Pi_{ \pm}^{12}=-\Pi_{ \pm}^{21}$. One can also decompose it into four parts with respect to $z$-component of spin, e.g., $s_{z}$, in the rest frame [29]:

$$
\begin{aligned}
\Pi_{ \pm}^{\mu \nu}(q)= & \Pi_{ \pm}^{s_{z}=+1} \epsilon_{1}^{\star, \mu} \epsilon_{1}^{\nu}+\Pi_{ \pm}^{s_{z}=-1} \epsilon_{2}^{\star, \mu} \epsilon_{2}^{\nu}+\Pi_{ \pm}^{s_{z}=0} b^{\mu} b^{\nu} \\
& +\Pi_{ \pm}^{u} u^{\mu} u^{\nu}
\end{aligned}
$$

where $u^{\mu}=(1,0,0,0)$ is the four momentum in the rest frame and spin projection operators are introduced: 
$\epsilon_{1}^{\mu}=\frac{1}{\sqrt{2}}(0,1, i, 0), \quad \epsilon_{2}^{\mu}=\frac{1}{\sqrt{2}}(0,1,-i, 0), \quad b^{\mu}=(0,0,0,1)$.

It is worthy to note that the last term $\Pi_{ \pm}^{u}$ corresponds to unphysical component of charged rho polarization function. Meanwhile, the charged rho propagator is written as:

$$
\begin{aligned}
D_{ \pm}^{\mu \nu}= & D_{ \pm}^{s_{z}=+1} \epsilon_{1}^{\star, \mu} \epsilon_{1}^{\nu}+D_{ \pm}^{s_{z}=-1} \epsilon_{2}^{\star, \mu} \epsilon_{2}^{\nu}+D_{ \pm}^{s_{z}=0} b^{\mu} b^{\nu} \\
& +D_{ \pm}^{u} u^{\mu} u^{\nu} .
\end{aligned}
$$

As a consequence, we obtain the propagator for each component:

$$
D_{ \pm}^{s_{z}}(q)=\frac{2 G_{V}}{1+2 G_{V} \Pi_{ \pm}^{s_{z}}(q)}
$$

and the mass for each component is determined by solving following equations:

$$
\begin{aligned}
1+2 G_{V} \Pi_{ \pm}^{s_{z}=+1}\left(q^{2}=m_{\rho^{ \pm}, s_{z}=+1}\right) & =1+2 G_{V}\left(\Pi_{ \pm}^{11}-i \Pi_{ \pm}^{12}\right) \\
& =0
\end{aligned}
$$

$$
\begin{aligned}
1+2 G_{V} \Pi_{ \pm}^{s_{z}=-1}\left(q^{2}=m_{\rho^{ \pm}, s_{z}=-1}\right) & =1+2 G_{V}\left(\Pi_{ \pm}^{11}+i \Pi_{ \pm}^{12}\right) \\
& =0
\end{aligned}
$$

$$
1+2 G_{V} \Pi_{ \pm}^{s_{z}=0}\left(q^{2}=m_{\rho^{ \pm}, s_{z}=0}\right)=1+2 G_{V} \Pi_{ \pm}^{33}=0 .
$$

For neutral $\rho$, the matrix of one loop polarization function is:

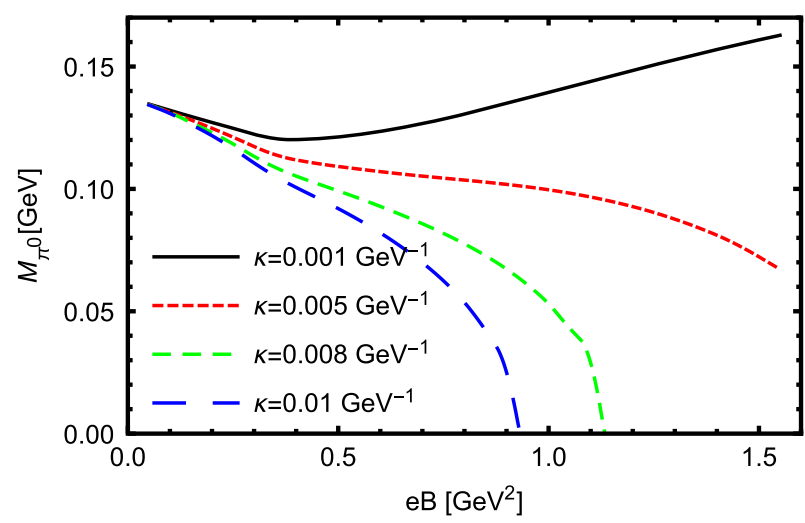

(a)

$$
\Pi_{0}^{\mu \nu}=\left(\begin{array}{cccc}
\Pi_{0}^{00} & 0 & 0 & 0 \\
0 & \Pi_{0}^{11} & 0 & 0 \\
0 & 0 & \Pi_{0}^{22} & 0 \\
0 & 0 & 0 & \Pi_{0}^{33}
\end{array}\right),
$$

where $\Pi_{0}^{11}=\Pi_{0}^{22}$, and the gap equations for neutral rho with $s_{z}= \pm 1,0$ are given by:

$$
\begin{aligned}
& 1+2 G_{V} \Pi_{0}^{s_{z}= \pm 1}\left(q^{2}=m_{\rho^{0}, s_{z}= \pm 1}\right)=1+2 G_{V} \Pi_{0}^{11}=0 \\
& 1+2 G_{V} \Pi_{0}^{s_{z}=0}\left(q^{2}=m_{\rho^{0}, s_{z}=0}\right)=1+2 G_{V} \Pi_{0}^{33}=0 .
\end{aligned}
$$

For neutral pion and rho, the Schwinger phase in quarkantiquark loop cancels out while it does not for charged pion and rho, leading a more complicated calculation. In this paper, we ignore the Schwinger phase and only consider the translation invariant part $\widetilde{S}$.

We present numerical results for the masses of pion and rho at zero temperature, where a soft cutoff is applied. The neutral and charged pion masses as functions of magnetic field are shown in Fig. 7. It is found that the presence of AMM reduces neutral pion mass $M_{\pi^{0}}$ significantly, and $M_{\pi^{0}}$ is very sensitive to the strength of AMM coupling, as shown in Fig. 7(a). Without AMM, neutral pion mass decreases slightly as magnetic field increases then it increases after an inflection point. When an appropriate AMM is applied, the previous inflection point disappears. $M_{\pi^{0}}$ continuously decreases and reaches zero at a critical magnetic field point $e B_{c}$. And as $\kappa$ increases, $e B_{c}$ decreases, which indicates a quick decline of $M_{\pi^{0}}$ as magnetic field increases. When $\kappa=0.005 \mathrm{GeV}^{-1}, e B_{c}$ is larger than $1.5 \mathrm{GeV}^{2}$; for $\kappa=0.008 \mathrm{GeV}^{-1}, e B_{c} \sim$ $1.15 \mathrm{GeV}^{2}$ and $e B_{c} \sim 0.95 \mathrm{GeV}^{2}$ for $\kappa=0.01 \mathrm{GeV}^{-1}$. It has been studied in many works $[32,33,36,37]$ that magnetic field enhances charged pion masses $M_{\pi^{ \pm}}$, as shown in

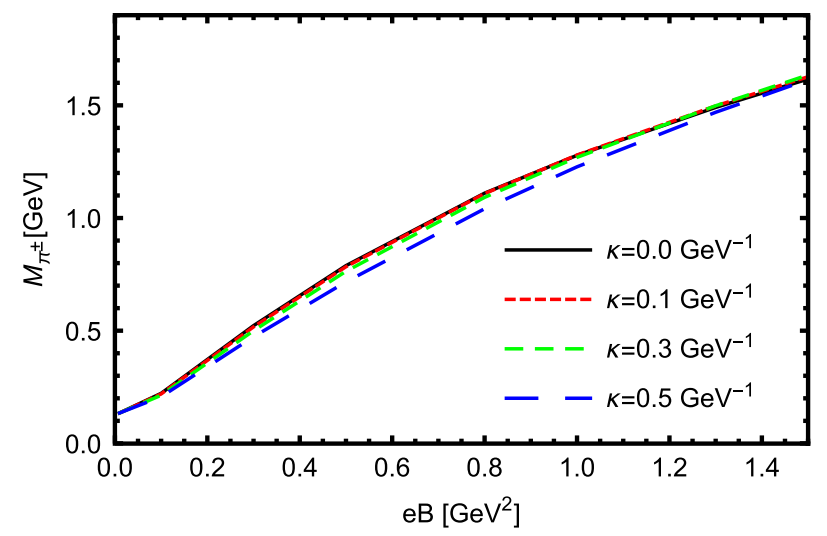

(b)

FIG. 7. (a) Neutral and (b) charged pion mass as functions of the magnetic field with different constant $\kappa$. 


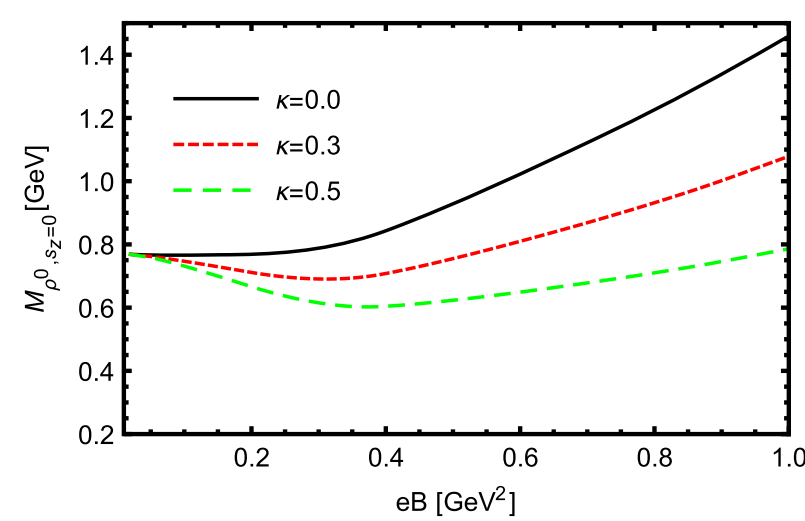

(a)

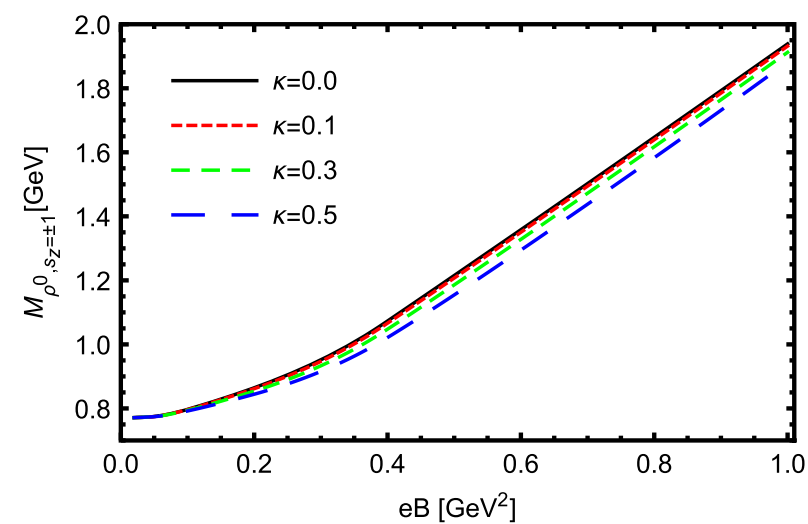

(b)

FIG. 8. Mass of neutron rho with (a) $s_{z}=0$ and (b) $s_{z}= \pm 1$ as a function of magnetic field with different constant $\kappa$.

Fig. 7(b). $M_{\pi^{ \pm}}$increase with magnetic field in both zero and nonzero AMM cases. Similar to neutral pion, AMM also reduces charged pion mass, however, the modification from AMM is too slight to be noticed. Besides, comparing to the results of neutral pion with the charged pion, it is seen that they exhibit different sensitivities to the AMM. A very small $\kappa$ of AMM can flip the behavior of neutral mass in the region of $e B>0.4 \mathrm{GeV}^{2}$ [dashed blue line shown

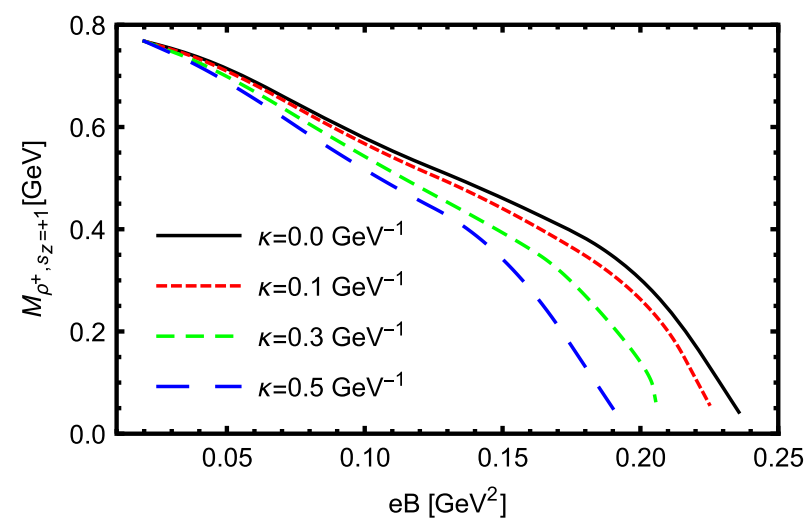

(a) in Fig. 7(a)] from increasing to decreasing along the magnetic field, while the behavior of charged pion mass is hardly changed even $\kappa=0.5 \mathrm{GeV}^{-1}$ [dashed blue line shown in Fig. 7(b)] at the range of magnetic field $0<e B<1.5 \mathrm{GeV}^{2}$.

Next, let us consider the effect of AMM on neutral rho mass with different spin component $s_{z}$, which is shown in Fig. 8. In the case of $\kappa=0$, all rho mass with

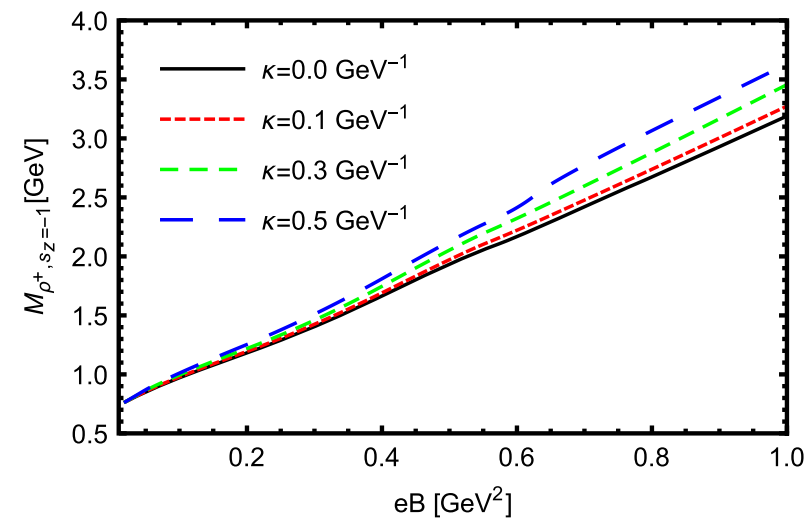

(b)

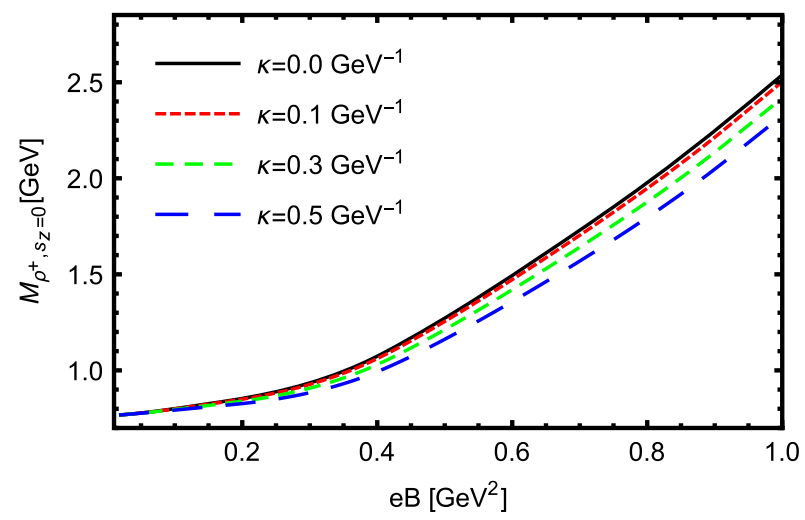

(c)

FIG. 9. Mass of charged rho with (a) $s_{z}=+1,(\mathrm{~b}) s_{z}=-1$ and (c) $s_{z}=0$ as a function of magnetic field with different constant $\kappa$. 
three different $s_{z}$ increase with magnetic field, while the presence of AMM reduces neutral rho mass $M_{\rho^{0}}$ regardless its spin. However AMM can reduce mass of neutral rho with $s_{z}=0$ significantly while the mass of neutral rho with $s_{z}= \pm 1$ is reduced slightly. Besides, when $\kappa \geq 0.7 \mathrm{GeV}^{-1}$, $M_{\rho^{0}}\left(s_{z}=0\right)$ decreases with magnetic field, opposite to the zero AMM case.

For charged rho we only consider it with positive charge, and the numerical results are shown in Fig. 9. It is observed that AMM interaction reduces $M_{\rho^{+}}\left(s_{z}=+1\right)$ and $M_{\rho^{+}}\left(s_{z}=0\right)$ while increases $M_{\rho^{+}}\left(s_{z}=-1\right)$. For positive charged rho with $s_{z}=+1$, its mass $M_{\rho^{+}}\left(s_{z}=+1\right)$ decreases with magnetic in both zero and non-zero AMM cases, and declines to zero at a critical magnetic field $e B_{c}$, which is similar to neutral pion, $e B_{c}$ decreasing as $\kappa$ growing. It indicates that the vacuum has been more strongly polarized when consider the AMM of quarks. For rho with $s_{z}=-1$ and $s_{z}=0$, the modifications of masses induced by AMM can be ignored compared to their masses.

To be compatible with the different values of the proton and neutron anomalous magnetic moments $k_{p}=1.79$, $k_{n}=-1.91$, we also calculate the quark, pion and rho meson masses by taking two different values of $\kappa$ for $u$ quark and $d$ quark, by taking two sets value of $\kappa$ used in [51]. The set I $\kappa 1$ with $\kappa_{u}=0.00995, \kappa_{d}=0.07975$ and the set II $\kappa 2$ with $\kappa_{u}=0.29016, \kappa_{d}=0.35986$. We find that the results of quark, pion, and rho meson masses behave qualitatively the same as those in the case of taking small constant $\kappa$ in the current paper, so we will not show the detailed numerical figures.

\section{DISCUSSION AND CONCLUSION}

In this paper, we systematically investigate the effects of the anomalous magnetic moments of quarks in the magnetized QCD matter by employing the two-flavor NambuJona-Lasinio model. We have computed the magnetic susceptibility, the inverse magnetic catalysis around the critical temperature and the neutral/charged pion and rho meson spectra under magnetic fields. The dynamical AMM of quarks is presented by the term $\bar{\psi} \kappa_{f} q_{f} \sigma^{\mu \nu} F_{\mu \nu} \psi$ in the Lagrangian, whose coupling with magnetic field causes Zeeman splitting in the energy dispersion of quarks then changes the magnetism properties and meson spectra under magnetic fields. It is found that through including the AMM coupling cannot fully explain the quantities from lattice simulation of the magnetized matter. Nevertheless, it partially reproduces the lattice results. Our results are summarized as follows:

(1) Quark's AMM reduces the dynamical quark mass which is consistent with the dispersion relation Eq. (21), a proper choice of $\kappa \sim \sigma$ can produce the inverse magnetic catalysis around the critical temperature $T_{c}$.

(2) The neutral pion mass is very sensitive to the AMM, it decreases with magnetic field quickly, and reaches to zero at a critical magnetic field $e B_{c}$ where $e B_{c}$ decreases as $\kappa$ increases. On the other hand, the charged pion mass almost does not change with $\kappa$, which shows a nontrivial behavior, i.e., linearly increases with $B$ in the small region and then saturates for strong $B$. The lattice calculation in [26] shows that the neutral pion mass decreases with the magnetic field and then saturates at about $0.6 M_{\pi^{0}}(e B=0)$ for $e B>1 \mathrm{GeV}^{2}$, and the charged pion mass straightly grows till $e B \sim 0.6 \mathrm{GeV}^{2}$ and then decreases with magnetic field.

(3) It is more complicated for $\rho$ because different spin components $s_{z}$ of rho behave differently. It is observed that AMM reduces the mass of neutral rho meson for three spin components $s_{z}$, reduces the mass of $s_{z}=+1,0$ components of charged rho but enhances the mass of $s_{z}=-1$ state for charged rho. Similar to pion, the neutral rho meson is more sensitive to the AMM coupling than the charged ones. AMM interaction reduces the masses of neutral rho with $s_{z}=0$ and $s_{z}= \pm 1$, however, the former one changes significantly while the latter one only shows a slight modification, which can be neglected comparing to their masses. Besides, for $\kappa \geq 0.7 \mathrm{GeV}^{-1}, M_{\rho^{0}}\left(s_{z}=0\right)$ decreases continuously with magnetic field, while it increases monotonously at zero AMM case. For charged rho with spin components $s_{z}=+1$, their masses decrease with magnetic filed and reach to zero at a critical magnetic field $e B_{c}$. Comparing with $\kappa=0$, its mass also is smaller for larger $\kappa$ at given finite $B$, which indicates that the magnetized matter is more easily to be polarized after including the AMM term. Besides, AMM coupling slightly reduces and enhances $M_{\rho^{+}}\left(s_{z}=0\right)$ and $M_{\rho^{+}}\left(s_{z}=-1\right)$, respectively.

(4) The magnetic susceptibility of the magnetized QCD matter cannot be explained by including the AMM of quarks. It is found that at low temperature the magnetic susceptibility can be either positive (paramagnetism) or negative(diamagnetism) with different values of AMM.

In summary, taking into account of the AMM of quarks cannot fully describe lattice results on magnetized matter and meson spectra. More studies and other mechanisms need to be investigated in the future.

\section{ACKNOWLEDGMENTS}

This work is supported in part by the National Natural Science Foundation of China (NSFC) Grants No. 11735007, No. 11725523, and Chinese Academy of Sciences under Grant No. XDPB09, the start-up funding from University of Chinese Academy of Sciences (UCAS), and the Fundamental Research Funds for the Central Universities. 
[1] T. A. Mihara et al., Nature (London) 346, 250 (1990); R. C. Duncan and C. Thompson, Astrophys. J. 392, L9 (1992); A. Lyne and F. Graham-Smith, Pulsar Astronomy (Cambridge University Press, Cambridge, England, 2005).

[2] T. Tatsumi, AIP Conf. Proc. 847, 171 (2006).

[3] K. Kiuchi, P. Cerd-Durn, K. Kyutoku, Y. Sekiguchi, and M. Shibata, Phys. Rev. D 92, 124034 (2015).

[4] L. Baiotti and L. Rezzolla, Rep. Prog. Phys. 80, 096901 (2017).

[5] V. Skokov, A. Y. Illarionov, and V. Toneev, Int. J. Mod. Phys. A 24, 5925 (2009).

[6] W.-T. Deng and X.-G. Huang, Phys. Rev. C 85, 044907 (2012).

[7] T. Vachaspati, Phys. Lett. B 265, 258 (1991).

[8] J. O. Andersen, W. R. Naylor, and A. Tranberg, Rev. Mod. Phys. 88, 025001 (2016).

[9] V. A. Miransky and I. A. Shovkovy, Phys. Rep. 576, 1 (2015).

[10] X. G. Huang, Rep. Prog. Phys. 79, 076302 (2016).

[11] D. E. Kharzeev, J. Liao, S. A. Voloshin, and G. Wang, Prog. Part. Nucl. Phys. 88, 1 (2016).

[12] A. Bzdak, S. Esumi, V. Koch, J. Liao, M. Stephanov, and N. Xu, Phys. Rep. 853, 1 (2020).

[13] D. Kharzeev and A. Zhitnitsky, Nucl. Phys. A797, 67 (2007).

[14] D. E. Kharzeev, L. D. McLerran, and H. J. Warringa, Nucl. Phys. A803, 227 (2008).

[15] K. Fukushima, D. E. Kharzeev, and H. J. Warringa, Phys. Rev. D 78, 074033 (2008).

[16] D. E. Kharzeev and D. T. Son, Phys. Rev. Lett. 106, 062301 (2011).

[17] S. P. Klevansky and R. H. Lemmer, Phys. Rev. D 39, 3478 (1989).

[18] K. G. Klimenko, Teor. Mat. Fiz. 89, 211 (1991) [Theor. Math. Phys. 89, 1161 (1991)].

[19] V. P. Gusynin, V. A. Miransky, and I. A. Shovkovy, Nucl. Phys. B462, 249 (1996); V. P. Gusynin, V. A. Miransky, and I. A. Shovkovy, Nucl. Phys. B563, 361 (1999).

[20] G. S. Bali, F. Bruckmann, G. Endrodi, Z. Fodor, S. D. Katz, S. Krieg, A. Schafer, and K. K. Szabo, J. High Energy Phys. 02 (2012) 044.

[21] G. S. Bali, F. Bruckmann, G. Endrodi, Z. Fodor, S. D. Katz, and A. Schafer, Phys. Rev. D 86, 071502 (2012).

[22] G. S. Bali, F. Bruckmann, G. Endrodi, F. Gruber, and A. Schaefer, J. High Energy Phys. 04 (2013) 130.

[23] K. Fukushima and Y. Hidaka, Phys. Rev. Lett. 110, 031601 (2013).

[24] J. Chao, P. Chu, and M. Huang, Phys. Rev. D 88, 054009 (2013).

[25] E. J. Ferrer, V. de la Incera, and X. J. Wen, Phys. Rev. D 91, 054006 (2015).

[26] H. T. Ding, S. T. Li, S. Mukherjee, A. Tomiya, and X. D. Wang, Proc. Sci., LATTICE2019 (2020) 250 [arXiv:2001.05322].

[27] G. S. Bali, F. Bruckmann, M. Constantinou, M. Costa, G. Endrodi, S. D. Katz, H. Panagopoulos, and A. Schafer, Phys. Rev. D 86, 094512 (2012).

[28] G. S. Bali, G. Endrdi, and S. Piemonte, J. High Energy Phys. 07 (2020) 183.
[29] H. Liu, L. Yu, and M. Huang, Phys. Rev. D 91, 014017 (2015).

[30] H. Liu, L. Yu, and M. Huang, Chin. Phys. C 40, 023102 (2016).

[31] H. Liu, X. Wang, L. Yu, and M. Huang, Phys. Rev. D 97, 076008 (2018).

[32] Z. Wang and P. Zhuang, Phys. Rev. D 97, 034026 (2018).

[33] S. Mao, Phys. Rev. D 99, 056005 (2019).

[34] S. S. Avancini, W. R. Tavares, and M. B. Pinto, Phys. Rev. D 93, 014010 (2016).

[35] S. S. Avancini, R. L. S. Farias, M. Benghi Pinto, W. R. Tavares, and V. S. Timteo, Phys. Lett. B 767, 247 (2017).

[36] M. Coppola, D. Gmez Dumm, and N. Scoccola, Phys. Lett. B 782, 155-161 (2018).

[37] M. Coppola, D. Gomez Dumm, S. Noguera, and N. Scoccola, Phys. Rev. D 100, 054014 (2019).

[38] S. Fayazbakhsh, S. Sadeghian, and N. Sadooghi, Phys. Rev. D 86, 085042 (2012).

[39] S. Fayazbakhsh and N. Sadooghi, Phys. Rev. D 88, 065030 (2013).

[40] S. P. Klevansky, Rev. Mod. Phys. 64, 649 (1992).

[41] Y. B. He, J. Hufner, S. P. Klevansky, and P. Rehberg, Nucl. Phys. A630, 719 (1998).

[42] P. Rehberg and S. P. Klevansky, Ann. Phys. (N.Y.) 252, 422 (1996).

[43] J. S. Schwinger, Phys. Rev. 82, 664 (1951).

[44] Y. Hidaka and A. Yamamoto, Phys. Rev. D 87, 094502 (2013).

[45] M. A. Andreichikov, B. O. Kerbikov, V. D. Orlovsky, and Y. A. Simonov, Phys. Rev. D 87, 094029 (2013).

[46] G. S. Bali, B. B. Brandt, G. Endrdi, and B. Glle, Phys. Rev. D 97, 034505 (2018).

[47] E. J. Ferrer, V. de la Incera, I. Portillo, and M. Quiroz, Phys. Rev. D 89, 085034 (2014).

[48] L. Chang, Y. Liu, and C. D. Roberts, Phys. Rev. Lett. 106, 072001 (2011).

[49] E. J. Ferrer and V. de la Incera, Phys. Rev. Lett. 102, 050402 (2009).

[50] S. Mao and D. H. Rischke, Phys. Lett. B 792, 149 (2019).

[51] J. Mei and S. Mao, Phys. Rev. D 102, 114035 (2020).

[52] E. J. Ferrer and V. de la Incera, Nucl. Phys. B824, 217 (2010).

[53] P. J. A. Bicudo, J. E. F. T. Ribeiro, and R. Fernandes, Phys. Rev. C 59, 1107 (1999).

[54] S. Fayazbakhsh and N. Sadooghi, Phys. Rev. D 90, 105030 (2014).

[55] M. Strickland, V. Dexheimer, and D. P. Menezes, Phys. Rev. D 86, 125032 (2012).

[56] N. Chaudhuri, S. Ghosh, S. Sarkar, and P. Roy, Phys. Rev. D 99, 116025 (2019).

[57] E. J. Ferrer, V. de la Incera, D. Manreza Paret, A. Prez Martnez, and A. Sanchez, Phys. Rev. D 91, 085041 (2015).

[58] N. Chaudhuri, S. Ghosh, S. Sarkar, and P. Roy, Eur. Phys. J. A 56, 213 (2020).

[59] S. Ghosh, N. Chaudhuri, S. Sarkar, and P. Roy, Phys. Rev. D 101, 096002 (2020).

[60] J. Chao, Y. X. Liu, and L. Chang, arXiv:2007.14258. 